\title{
A Transient Elevated Irisin Blood Concentration in Response to Prolonged, Moderate Aerobic Exercise in Young Men and Women
}

Authors

Affiliations

\author{
R. R. Kraemer ${ }^{1}$, P. Shockett ${ }^{2}$, N. D. Webb ${ }^{1}$, U. Shah ${ }^{3}$, V. D. Castracane ${ }^{3}$
}

${ }^{1}$ Department of Kinesiology and Health Studies, Southeastern Louisiana University, Hammond, LA, USA ${ }^{2}$ Department of Biological Sciences, Southeastern Louisiana University, Hammond, LA, USA ${ }^{3}$ Department of Obstetrics and Gynecology, Texas Tech University Health Sciences Center at the Permian Basin, Odessa, TXUSA

\section{Key words}

myokine

muscle contraction

- FNDC5 expression

- glucoregulation received 29.06.2013

accepted 26.08.2013

\section{Bibliography \\ DOI http://dx.doi.org/ 10.1055/s-0033-1355381 \\ Published online: \\ September 23, 2013 \\ Horm Metab Res 2014; \\ 46: 150-154 \\ (c) Georg Thieme Verlag KG \\ Stuttgart · New York \\ ISSN 0018-5043}

\section{Correspondence}

Dr. $\boldsymbol{R}$. R. Kraemer, FACSM

Department of Kinesiology and Health Studies

Southeastern Louisiana

University

SLU10845

Hammond

LA

USA

Tel.: + 1/985/549 2132

Fax: + 1/985/5495119

rkraemer@selu.edu

\section{Abstract \\ $\nabla$}

Irisin, a newly discovered, PGC- $1 \alpha$ dependent myokine, has recently been shown to increase in circulation in response to sprint exercise. This study examined the effect of prolonged exercise on irisin concentrations in young men $(n=7)$ as well as in young women $(n=5)$ during different stages of the menstrual cycle. Seven young men completed $90 \mathrm{~min}$ of treadmill exercise at $60 \%$ of $\mathrm{VO}_{2} \max$ and a resting control trial. Five women completed the same exercise protocol in two different trials: during the early follicular phase and mid-luteal phase of the menstrual cycle. Blood samples were collected and analyzed for irisin concentrations immediately before exercise,

\section{Introduction}

$\nabla$

Expression of the transcriptional co-activator, PPAR- $\gamma$ co-activator- $1 \alpha$ (PGC- $1 \alpha)$ is increased in skeletal muscle in response to exercise [1]. In 2010 , Bostrom et al. described a newly discovered, PGC- $1 \alpha$ dependent myokine, named irisin [2]. The investigators demonstrated that PGC- $1 \alpha$ stimulates expression of the membrane protein FNDC5, which is cleaved and released from muscle as the hormone irisin [2]. Irisin appears to act on white adipose tissue to increase the expression of uncoupling protein 1 (UCP1), found in mitochondria (typically of brown fat) and increases thermogenesis [2]. This is characteristic of brown fat, which has greater UCP1 expression and expends more calories than white fat. Thus, irisin has been shown to induce the "browning" of white fat and increase the ability of mitochondria in white adipose tissue to burn more stored fat. Specifically, white fat depots contain some "beige fat" cells that have low expression of UCP1, but cyclic AMP stimulation will increase UCP1 expression in these cells [3]. Moreover, these cells have a different pattern of at 54 and $90 \mathrm{~min}$ of exercise, and at $20 \mathrm{~min}$ of recovery (R20). Findings revealed that by $54 \mathrm{~min}$ of a 90 min treadmill exercise protocol at $60 \%$ of $\mathrm{VO}_{2} \mathrm{max}$, irisin concentrations significantly increased $20.4 \%$ in young men and $20.3 \%$ as well as $24.6 \%$ in young women during the early follicular and mid-luteal phases of the menstrual cycle, respectively. However, by 90 min of exercise as well as R20, irisin concentrations were no longer elevated. Stage of the menstrual cycle did not affect responses in young women. Findings indicate that prolonged aerobic exercise produces a transient increase in irisin concentrations during the first hour of exercise for both genders and suggest that this form of moderate exercise may be helpful in improving fat metabolism. gene expression than brown or white fat and are very sensitive to stimulation by irisin [3]. Bostrom et al. [2] also presented data indicating that 1 ) irisin stimulates mitochondrial biogenesis, leading to better fat metabolism, 2) small increases in circulating irisin increase caloric expenditure in mice without extra movement, and 3) preliminary experiments suggest 3 weeks of swim training in mice and 10 weeks of endurance training in humans increases resting irisin concentrations [2].

A recent investigation by Huh et al. [4] demonstrated that there were relationships between irisin and anthropometric measures (e.g., biceps brachii circumference, BMI) as well as hormone and metabolite concentrations (e.g., insulin, IGF1 , glucose). These investigators also reported evidence of elevated circulating irisin concentration $30 \mathrm{~min}$ following acute sprinting exercise before 8 weeks of training, but not after the training. There are no studies that have investigated acute responses of irisin to moderate aerobic exercise in humans. Understanding how exercise intensity and duration stimulate irisin release from muscle is important in that subsequent data 
Table 1 Descriptive data of subjects.

\begin{tabular}{|lcc|} 
Measure & Males $(\mathbf{n}=\mathbf{7})$ Mean \pm SD & Females $(\mathbf{n}=\mathbf{5})$ Mean \pm SD \\
\hline Age & $22.71 \pm 1.6$ years & $23.8 \pm 4.7$ years \\
\hline Height & $176.71 \pm 7.61 \mathrm{~cm}$ & $161.8 \pm 6.83 \mathrm{~cm}$ \\
Weight & $71.7 \pm 9.43 \mathrm{~kg}$ & $67.4 \pm 14.4 \mathrm{~kg}$ \\
$\mathrm{BMl}$ & $24.29 \pm 2.94 \mathrm{~kg} / \mathrm{m}^{2}$ & $25.2 \pm 4.97 \mathrm{~kg} / \mathrm{m}^{2}$ \\
$\mathrm{VO}_{2} \max$ & $55.77 \pm 9.8 \mathrm{ml} \cdot \mathrm{kg}^{-1} \cdot \mathrm{min}^{-1}$ & $34.8 \pm 6.68 \mathrm{ml} \cdot \mathrm{kg}^{-1} \cdot \mathrm{min}^{-1}$ \\
\hline
\end{tabular}

could be used to develop exercise regimens for improving fat metabolism and curbing diseases that occur with obesity or genetic predisposition, including heart disease and type II diabetes $[5,6]$.

We have conducted 2 experiments to determine the effects of exercise on plasma irisin concentrations in humans. First, the effects of continuous treadmill running on irisin concentrations in young men exercising for $90 \mathrm{~min}$ were compared to responses from a resting control trial. Samples from these subjects were used in a previous study to investigate glucoregulatory hormone and metabolite responses to prolonged exercise [7]. Second, since it was recently demonstrated that circulating irisin correlated significantly with insulin, glucose, and ghrelin [4], and since insulin has been shown to be affected by estradiol [8] we determined irisin responses to prolonged exercise in young women during early follicular and mid-luteal phases of their menstrual cycle.

We hypothesized that irisin concentrations would increase over time in response to longer exercise durations in the young men and women, due to a progressively greater amount of muscle contraction. Since it has recently been determined that irisin is related to $17-\beta$ estradiol concentrations [8], we hypothesized that irisin would increase to a greater degree in the mid-luteal phase of the menstrual cycle than in the early follicular phase.

\section{Materials and Methods}

$\nabla$

\section{Experiment 1}

Seven healthy, young male volunteers previously participated in a study to determine the effects of prolonged exercise on glucoregulatory function [7]. In the present study, we examined irisin responses to $90 \mathrm{~min}$ of treadmill exercise in those subjects (see Table 1 for descriptive data). After giving informed consent, subjects completed a medical history questionnaire and 3-day food record to meet the criteria for the study: 1 ) between the ages of 18 and 35 years, 2) not taking any prescription medications, 3) no history of cardiovascular or metabolic disease, and 4) no adherence to a diet that would affect metabolic responses to exercise. Subjects were all considered physically active and completed at least a regular weekly minimum of 3-4 aerobic workouts per week for $30-60$ min per session. Subjects completed a preliminary trial followed by an experimental (exercise) trial and a control (resting) trial in counterbalanced manner with 1 month between trials.

For the preliminary trial, all subjects completed a graded treadmill exercise test to exhaustion using the Kraemer Protocol [7] and a metabolic cart (ParvoMedics 2400, Sandy UT) to determine $\mathrm{VO}_{2} \mathrm{max}$, establish by standardized criteria [7]. For the exercise and control trials, subjects reported to the lab at 8:00 $\mathrm{AM}$ after consuming a liquid meal (Ensure Plus ${ }^{\mathrm{TM}}$ ) at 7:00 AM. The liquid meal prevented fasting as well as the $700-900 \mathrm{kcal}$ expenditure during exercise from contributing to the metabolic and endocrine responses. After IV catheter insertion, blood samples ( $28 \mathrm{ml} /$ samples) were collected at rest, during and following treadmill exercise at $60 \%$ of $\mathrm{VO}_{2} \mathrm{max}$ for $90 \mathrm{~min}$. In the control trial, subjects remained in a seated position, but followed identical procedures as the exercise trial excluding the exercise and metabolic analysis. Irisin concentrations were determined for pre- (0 min), during (+54 min and $90 \mathrm{~min}$ ), and following (R20 min) exercise or control. Hematocrit was determined using the microhematocrit method and hemoglobin was determined from whole blood using an enzymatic, colorimetric assay (Pointe Scientific, Canton, MI, USA). Plasma volume shifts were determined using the method of Dill and Costill [9].

\section{Experiment 2}

After giving informed consent, 5 healthy, female subjects previously participated in a study to determine the effects of prolonged ( $90 \mathrm{~min}$ ) treadmill exercise on glucoregulatory function in different stages of the menstrual cycle [10] (see $\bullet$ Table 1 for descriptive data). For the present study we determined irisin responses to prolonged exercise in different stages of the menstrual cycle. Subjects were screened to ensure they met the following criteria to participate in the study: 1) normal menstrual cycles (range: 26-34 d; 2) between the ages of 20 and 35 years; 3) no past history of metabolic or cardiovascular diseases; 4) not taking any medications; and 5) recreationally trained for minimum of 3 previous months.

To establish menstrual cycle length and regularity, menstrual cycles were followed for 3 consecutive months before testing. Subjects completed a preliminary trial to determine $\mathrm{VO}_{2} \max$ and then were scheduled for testing in early follicular ( $d$ 3-7) and mid-luteal phases (d 20-22) of the menstrual cycle in a counter-balanced fashion. Estradiol and progesterone values were measured to ensure that the women were indeed in the mid-luteal or early follicular phase of their menstrual cycle [10]. For early follicular exercise (EFX) and mid-luteal exercise (MLX) trials, subjects reported to the lab after an overnight fast and an IV catheter was inserted and kept patent with a saline lock. Blood samples ( $28 \mathrm{ml} / \mathrm{sample}$ ) were collected at rest, as well as during and following treadmill exercise at $60 \%$ of $\mathrm{VO}_{2} \max$ for $90 \mathrm{~min}$. Blood samples for experiment 1 and 2 were drawn into $10 \mathrm{ml}$ EDTA tubes, immediately centrifuged at $4{ }^{\circ} \mathrm{C}$, separated after centrifugation, and stored at $-80^{\circ} \mathrm{C}$ until analysis. Irisin concentrations were determined for pre- $(0 \mathrm{~min})$, during $(+54 \mathrm{~min}$ and $90 \mathrm{~min}$ ), and following (R20 min) exercise.

Blood samples were analyzed for irisin by ELISA (Phoenix Pharmaceuticals, Burlingame, CA, USA). The ELISA was originally developed by Aviscera BioScience and recently sold through Phoenix Pharmaceuticals. The same ELISA was used in the recent study by Huh et al. [4] and was reported to be the most reliable among several commercially available kits. Interassay and intraassay coefficient of variation were 7.76 and 8.97, respectively. Sensitivity of the assay was $4.15 \mathrm{ng} / \mathrm{ml}$. None of the plasma samples assayed for this study approached this level of sensitivity.

\section{Statistics}

For the first experiment, a trial (exercise/control) $\times$ time $(0,+54,+90, \mathrm{R} 20)$ ANOVA was conducted. For the second experiment a trial $($ EFX/MLX) $\times$ time $(0,+54,+90$, R20) ANOVA was conducted. To compare irisin concentrations between men and women, a trial (male exercise, male control, female EFX, female $\operatorname{MLX}) \times$ time $(0,+54,+90$, R20) ANOVA was conducted. Depend- 
ent $t$-tests were applied where appropriate for post-hoc analyses.

\section{Results}

Findings for the first experiment revealed a significant time effect $[F(3,36)=5.28, p=0.004]$ and significant time $\times$ trial interaction $[F(3,36)=4.16, p=0.013]$. Post-hoc analyses revealed that during the exercise trial, irisin concentrations increased significantly by $20.4 \%$ from 0 to 54 min of treadmill exercise. From 54 to $90 \mathrm{~min}$, irisin concentrations tended to decline, but not significantly $(\mathrm{p}=0.074)$ compared to $54 \mathrm{~min}$; however, concentrations declined significantly from $54 \mathrm{~min}$ to $\mathrm{R} 20(\mathrm{p}=0.021)$. Pre-exercise concentrations were not significantly different compared to $90 \mathrm{~min}(\mathrm{p}=0.11$ ) and $\mathrm{R} 20$ ( $\mathrm{p}=0.7$ ) ( $\bullet$ Fig. 1). Plasma irisin levels were unchanged across time during the control trial. We also conducted a trial $\times$ time ANOVA and post-hoc analyses with irisin concentrations that were corrected for plasma volume shifts using the hematocrit and hemoglobin values [9] in the exercise and control trials. Results were similar with a time effect $[F(3,36)=5.55, p=0.003]$ and significant time $\times$ trial interaction $[F(3,36)=4.16, p=0.010]$ for irisin concentrations corrected for plasma volume shifts. Moreover, post-hoc analyses of corrected irisin concentrations revealed significance between the same time points for the exercise trial (pre and $54 \mathrm{~min}$; $54 \mathrm{~min}$ and $90 \mathrm{~min}$ ) and nonsignificance between the same time points for the exercise and control trials.

Results from the second experiment revealed a significant time effect $[F(3,24)=5.03, p=0.008]$ with no time $\times$ trial interaction $[F(3,24)=0.50, p=0.686]$ indicating a similar change over time in the MLX and EFX trials. Post-hoc analysis revealed significantly greater $(p<0.05)$ irisin concentrations at 54 min compared to resting values, regardless of menstrual cycle phase (early follicular or mid-luteal). Irisin concentrations rose $20.3 \%$ and $24.6 \%$ by $54 \mathrm{~min}$ compared to resting values in the early follicular and mid-luteal phases of the menstrual cycle, respectively, and were lower by $90 \mathrm{~min}$ of exercise and during recovery $(\mathrm{p}>0.05$ between 0 and $90 \mathrm{~min}$ as well as $0 \mathrm{~min}$ and R20, $\odot$ Fig. 2). We did not correct for plasma volume shifts in the second experiment since hemoglobin values were unavailable.

Comparison of irisin concentrations in men and women revealed a significant time effect $[F(3,60)=9.06, p=0.000]$ with no time $\times$ trial (male exercise trial, EFX trial, MLX trial) interaction $[F(3,60)=1.91, p=0.067]$.

\section{Discussion}

$\nabla$

This is the first study to determine the effects of prolonged exercise on irisin concentrations. It is also the first study to compare these responses in men and women. Consistent with one of our experimental hypotheses, the major finding was that by $54 \mathrm{~min}$ of prolonged, moderate-intensity aerobic exercise, irisin concentrations increased, but were no longer elevated by $90 \mathrm{~min}$ and significantly lower by $20 \mathrm{~min}$ of recovery. This response occurred in both healthy young men and women, and contrary to our hypothesis, there was no observed effect of the menstrual cycle on female irisin responses. This is the first study to report a transient increase in irisin responses to prolonged exercise in men and women, with no difference in responses between genders.

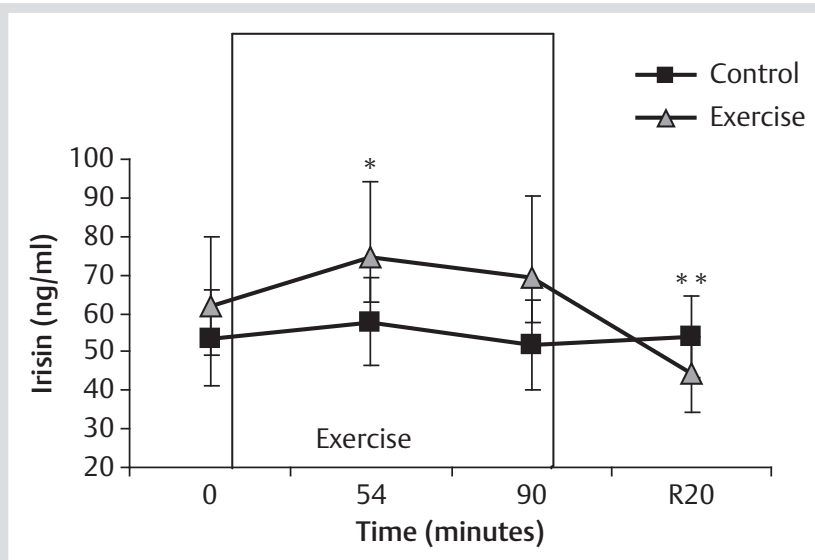

Fig. 1 Irisin responses to 90 min of treadmill exercise in 7 young men for the control and exercise trials. ${ }^{*}$ Significantly different than pre-exercise concentrations. ${ }^{*}$ Significantly different than 54 min concentrations.

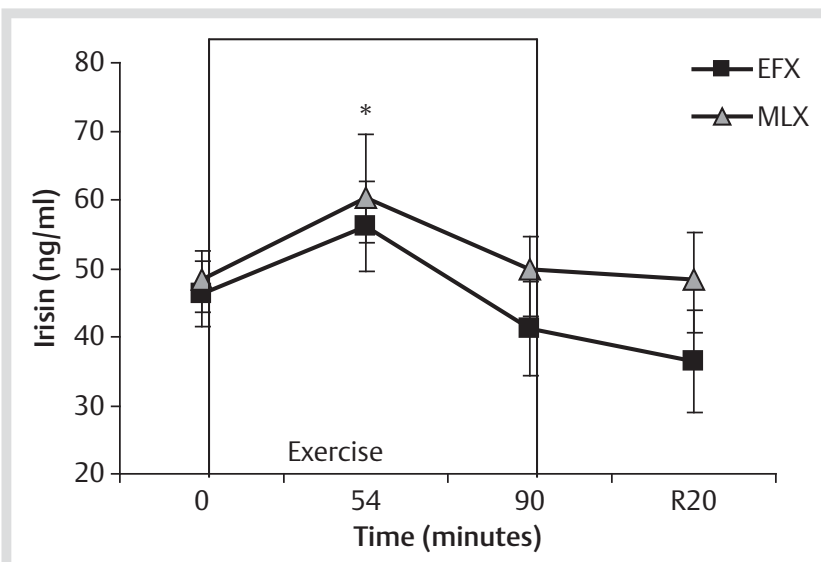

Fig. 2 Irisin responses to $90 \mathrm{~min}$ of treadmill exercise in 5 young women. EFX: early follicular phase; MLX: mid-luteal phase. ${ }^{*}$ Significantly different than pre-exercise concentrations.

There are several investigations that have reported PGC- $1 \alpha$ mRNA expression in skeletal muscle after acute exercise. However, these studies report increases in PGC- $1 \alpha$ well after the time point in which we report increases in circulating concentrations of irisin in response to exercise. Sriwijitkamol et al. [11] reported no change in PGC- $1 \alpha$ expression in muscle, after $40 \mathrm{~min}$ of cycling at both a low $\left(50 \% \mathrm{VO}_{2} \max \right)$ and moderate $\left(70 \% \mathrm{VO}_{2} \max \right)$ exercise intensity, but increases in leg muscle expression of PGC- $1 \alpha$ mRNA in subjects 150 min after cycling. Wang et al. $[12,13]$ also found increases in PGC- $1 \alpha$ mRNA expression in skeletal muscle $3 \mathrm{~h}$ after acute exercise. Here, we report transient increases in irisin concentrations after 54 min of continuous exercise, followed by reductions in irisin. These transient increases in circulating irisin did not occur at a time point in which exercise-induced increases in PGC- $1 \alpha$ would be expected to have occurred in muscle, and thus further work is warranted to determine the cause of increased irisin concentrations.

There are only 2 previous papers reporting changes in irisin concentrations after exercise, and neither of the studies determined acute effects of moderate aerobic exercise (a form of exercise widely used) on plasma irisin responses. The first study was reported by Bostrom et al. [2] in which 3 weeks of swim training resulted in a $65 \%$ increase in resting irisin concentrations in mice, and 10 weeks of endurance exercise training produced a 
2 -fold increase in resting irisin concentrations in 8 human subjects. The second investigation by Huh et al. [4] reported acute increases (ca. 18\%) in circulating irisin concentrations in subjects 30 min after completing 2 sets of 2, 80-m sprints with either $10 \mathrm{~s}$ or $1 \mathrm{~min}$ between sprints and $20 \mathrm{~min}$ between sets [14]. Using the information from the experimental design of that study, we have estimated the time from exercise onset to postexercise blood draw to be approximately $55 \mathrm{~min}$. Based on data from Sriwijitkamol et al. [11], PGC- $1 \alpha$ concentrations may not have increased in skeletal muscle to stimulate irisin release from muscle by that time. Thus, it is very plausible that the reported increase in irisin concentration in that study as well as the increase reported in the present study after $54 \mathrm{~min}$ of exercise was not stimulated by PGC- $1 \alpha$, but by other stimuli. Why irisin levels were no longer elevated at $+90 \mathrm{~min}$ and $20 \mathrm{~min}$ of recovery remains to be determined.

Interestingly, after subjects in the study by Huh et al. [2] completed 8 weeks of sprint training (first 4 weeks: 2 sets; second 4 weeks: 3 sets) irisin concentrations were measured in response to 3 sets of sprints. The investigators reported no acute change in irisin 8 weeks post-training, after completing 3 sets of $80-\mathrm{m}$ sprints with $20 \mathrm{~min}$ rest between sets. We have estimated the time from exercise onset to post-exercise blood draw to be approximately $75 \mathrm{~min}$. Whether the lack of an irisin response to the 3 set protocol was due to a training adaptation or to the timing of blood sampling is not known.

The present study provides the first evidence that prolonged aerobic exercise at a moderate intensity stimulates an acute, transient increase in irisin concentrations that could ultimately improve the metabolism of fat tissue. The acute increases in irisin after 54 min of steady-state exercise in both the women and the men, suggests no gender effect for irisin responses to exercise.

In addition to skeletal muscle, there are other tissue sources of irisin. Irisin has been shown to be expressed in cardiomyocytes and purkinje cells of the cerebellum [15]. Additionally, FNDC5 expression, the irisin precursor, was found to be greater in skeletal muscle of heart failure patients with greater functional (exercise) capacity $\left(\mathrm{VO}_{2} \max \right)$ than those with lower functional capacity [16]. Moreover, irisin has recently been shown to be expressed in fat tissue, although expression appears to be much less than in muscle tissue [17]. Due to its demonstrated signaling in adipose tissue, and its positive effect on fat metabolism, investigating irisin may help elucidate some of the mechanisms regarding development of type II diabetes and changes in metabolic flexibility [5]. Recent data indicate that irisin is lower in T2D patients and is related to 2-h plasma glucose concentrations [18]. Collectively, this work indicates the importance of this myokine.

The evidence from our experiments of transient, acute increases in irisin during moderate-intensity aerobic exercise suggests an important mechanism for aerobic-training-induced improvement in metabolic flexibility and fat metabolism that have previously been reported $[19,20]$. The increase in concentrations of irisin at +54 min declined by +90 min of steady-state exercise and were not significantly different than resting values in experiments 1 and 2, suggesting that irisin signaling from sustained, steady-state exercise occurs in the first hour of exercise. After correcting for plasma volume shifts during the exercise and control trials for the first experiment, we have demonstrated that changes in irisin concentrations at $54 \mathrm{~min}$ were significantly elevated at $54 \mathrm{~min}$ of exercise and did not change the statistical findings for the male subjects. Using hematocrit data available from the investigation of young women (experiment 2) [10], changes in plasma volume shifts for the MLX and EFX trials were estimated to be [mean \pm SD] $-3.0 \pm 6.09 \%$ from 0 min to +54 min and $-5.01 \pm 4.7 \%$ from 0 to +90 min. Thus, only small change in plasma volume occurred across these time points and the exercise protocol was similar for that of the men. Collectively this suggests that the $\approx 20 \%$ increase in irisin at +54 min of exercise for both men and women was not due to plasma volume shifts. More investigations are required to determine the precise exercise duration and intensity that will optimize irisin's effects. Interestingly, the percent increases in circulating irisin are slightly higher than those recently reported for acute responses to sprint exercise in untrained subjects [4], but no irisin change in response to sprinting was found after 8 weeks of training. With regard to the trained status of subjects in our experiments, the women were recreationally active but had not been completing a minimum number of aerobic workouts per week. The men, however, had been completing a minimum of 3-4 aerobic workouts of 30-60 min duration per week prior to the study. The level of cardiorespiratory fitness for females was moderately low $\left(\mathrm{VO}_{2} \mathrm{max} \approx 35 \mathrm{ml} / \mathrm{kg} / \mathrm{min}\right)$; however the male values were high $\left(\mathrm{VO}_{2} \mathrm{max} \approx 58 \mathrm{ml} / \mathrm{kg} / \mathrm{min}\right)$. Collectively, this indicates that the males were more trained than the females and that exercise elicited a similar increase in irisin concentrations in the men as those produced in the women who were not as well-trained. This suggests that training state did not affect the transient irisin responses to prolonged running at $60 \%$ of $\mathrm{VO}_{2}$ max of each subject. However, the response of adipose tissue to irisin may change with training and further studies regarding the effects of training on irisin expression and subsequent effect on adipose tissue are needed.

In conclusion, this study provides the first evidence that sustained treadmill exercise at a moderate intensity increases irisin concentrations in the first hour of exercise with values reduced by $90 \mathrm{~min}$ of exercise and further decline by $20 \mathrm{~min}$ of recovery. It is not known whether this transient increase in irisin concentration during the first hour of prolonged ( $90 \mathrm{~min})$ moderate aerobic exercise will have a subsequent effect on adipose tissue, thus further investigation is required. There appears to be no effect of gender or stage of the menstrual cycle on irisin responses to exercise, and additional studies with a larger number of female subjects is warranted to verify no effect of menstrual cycle phase. Irisin signaling in response to exercise may be an important mechanism to stimulate mitochondrial biogenesis and produce browning of fat to improve metabolism. Future work is needed to identify effects of different exercise protocols to optimize the possible beneficial effects of irisin.

\section{Acknowledgements}

$\nabla$

This work was supported by a Southeastern Louisiana University Faculty Enhancement Grant (2012-2013). We wish to thank the subjects for their participation in the study. We also wish to thank Adriana Flores-Villacrez for her work in the lab and for data management. This study is dedicated to Michelle Francois (1956-2012); her wonderful contributions to our lab will be missed. 


\section{Declaration of Interest}

$\nabla$

There is no conflict of interest that could be perceived as prejudicing the impartiality of the research reported.

\section{References}

1 Stepto NK, Benziane B, Wadley GD, Chibalin AV, Canny BJ, Eynon N, McConell GK. Short-term intensified cycle training alters acute and chronic responses of PGC1 $\alpha$ and Cytochrome C oxidase IV to exercise in human skeletal muscle. PLoS One 2012; 7: e53080

2 Boström P, Wu J, Jedrychowski MP, Korde A, Ye L, Lo JC, Rasbach KA, Boström EA, Choi JH, Long JZ, Kajimura S, Zingaretti MC, Vind BF, Tu $H$, Cinti S, Højlund K, Gygi SP, Spiegelman BM. A PGC1- $\alpha$-dependent myokine that drives brown-fat-like development of white fat and thermogenesis. Nature 2012; 481: 463-468

3 Wu J, Boström P, Sparks LM, Ye L, Choi JH, Giang AH, Khandekar M, Virtanen KA, Nuutila P, Schaart G, Huang K, Tu H, van Marken Lichtenbelt WD, Hoeks J, Enerbäck S, Schrauwen P, Spiegelman BM. Beige adipocytes are a distinct type of thermogenic fat cell in mouse and human. Cell 2012; 150: 366-376

4 Huh JY, Panagiotou G, Mougios V, Brinkoetter M, Vamvini MT, Schneider $B E$, Mantzoros CS. FNDC5 and irisin in humans: I. Predictors of circulating concentrations in serum and plasma and II. mRNA expression and circulating concentrations in response to weight loss and exercise. Metabolism 2012; 61: 1725-1738

5 Russell RD, Kraemer RR, Nelson AG. Metabolic dysfunction in diabetic offspring: deviations in metabolic flexibility. Med Sci Sports Exerc 2013; 45: 8-15

6 Smith MM, Minson CT. Obesity and adipokines: effects on sympathetic overactivity. J Physiol 2012; 590: 1787-1801

7 Kraemer RR, Francois MR, Sehgal K, Sirikul B, Valverde RA, Castracane $V D$. Amylin and selective glucoregulatory peptide alterations during prolonged exercise. Med Sci Sports Exerc 2011; 43: 1451-1456

8 Horton TJ, Miller EK, Glueck D, Tench K. No effect of menstrual cycle phase on glucose kinetics and fuel oxidation during moderate-intensity exercise. Am J Physiol Endocrinol Metab 2002; 282: E752-E762

9 Dill DB, Costill DL. Calculation of percentage changes in volumes of blood, plasma, and red cells in dehydration. J Appl Physiol 1974; 37: 247-248
10 Kraemer RR, Francois $M$, Webb ND, Worley JR, Rogers SN, Norman RL, Shah $U$, Castracane VD. No effect of menstrual cycle phase on glucose and glucoregulatory endocrine responses to prolonged exercise. Eur J Appl Physiol 2013; 113: 2401-2408

11 Sriwijitkamol A, Coletta DK, Wajcberg E, Balbontin GB, Reyna SM, Barrientes J, Eagan PA, Jenkinson CP, Cersosimo E, DeFronzo RA, Sakamoto $K$, Musi $N$. Effect of acute exercise on AMPK signaling in skeletal muscle of subjects with type 2 diabetes: a time-course and dose-response study. Diabetes 2007; 56: 836-848

12 Wang L, Mascher H, Psilander N, Blomstrand E, Sahlin K. Resistance exercise enhances the molecular signaling of mitochondrial biogenesis induced by endurance exercise in human skeletal muscle. J Appl Physiol 2011; 111: 1335-1344

13 Wang L, Psilander N, Tonkonogi M, Ding S, Sahlin K. Similar expression of oxidative genes after interval and continuous exercise. Med Sci Sports Exerc 2009; 41: 2136-2144

14 Saraslanidis P, Petridou A, Bogdanis GC, Galanis N, Tsalis G, Kellis S, Mougios $V$. Muscle metabolism and performance improvement after two training programmes of sprint running differing in rest interval duration. J Sports Sci 2011; 29: 1167-1174

15 Dun SL, Lyu RM, Chen YH, Chang JK, Luo JJ, Dun NJ. Neuroscience. Irisinimmunoreactivity in neural and non-neural cells of the rodent. Neuroscience 2013; 240: 155-162

16 Lecker SH, Zavin A, Cao P, Arena R, Allsup K, Daniels KM, Joseph J, Schulze $P C$, Forman DE. Expression of the irisin precursor FNDC5 in skeletal muscle correlates with aerobic exercise performance in patients with heart failure. Circ Heart Fail 2012; 5: 812-818

17 Moreno-Navarrete JM, Ortega F, Serrano M, Guerra E, Pardo G, Tinahones F, Ricart W, Fernández-Real JM. Irisin is expressed and produced by human muscle and adipose tissue in association with obesity and insulin resistance. J Clin Endocrinol Metab 2013; 98: E769-E778

18 Choi YK, Kim MK, Bae KH, Seo HA, Jeong JY, Lee WK, Kim JG, Lee IK, Park KG. Serum irisin levels in new-onset type 2 diabetes. Diabetes Res Clin Pract 2013; 100: 96-101

19 O'Hagan C, De Vito G, Boreham CA. Exercise prescription in the treatment of type 2 diabetes mellitus: current practices, existing guidelines and future directions. Sports Med 2013; 43: 39-49

20 Umpierre D, Ribeiro PA, Schaan BD, Ribeiro JP. Volume of supervised exercise training impacts glycaemic control in patients with type 2 diabetes: a systematic review with meta-regression analysis. Diabetologia 2013; 56: 242-251 